\title{
Presence and consequence of tooth periapical radiolucency in patients with cirrhosis
}

This article was published in the following Dove Press journal:

Hepatic Medicine: Evidence and Research

13 September 2016

Number of times this article has been viewed

\section{Lea Ladegaard Grønkjær' \\ Palle Holmstrup ${ }^{2}$ \\ Søren Schou ${ }^{3}$ \\ Kristoffer Schwartz ${ }^{4}$ \\ Johanne Kongstad ${ }^{2}$ \\ Peter Jepsen ${ }^{1,5}$ \\ Hendrik Vilstrup' \\ 'Department of Hepatology and Gastroenterology, Aarhus University \\ Hospital, Aarhus, ${ }^{2}$ Section of Periodontology, Microbiology, and Community Dentistry, Department of Odontology, Faculty of Health and Medical Sciences, ${ }^{3}$ Section for Oral and Maxillofacial Surgery, Department of Odontology, Faculty of Health and Medical Sciences, University of Copenhagen, Copenhagen, ${ }^{4}$ Department of Oral and Maxillofacial Surgery, ${ }^{5}$ Department of Clinical Epidemiology, Aarhus University Hospital, Aarhus, Denmark}

Correspondence: Lea Ladegaard Grønkjær

Department of Hepatology and Gastroenterology, Aarhus University Hospital, Nørrebrogade 44, 8000 Aarhus C, Denmark

$\mathrm{Tel}+4526668 \mathrm{I} 84$

Fax +4578462853

Email lealad@rm.dk
Background: Periapical radiolucency is the radiographic sign of inflammatory bone lesions around the apex of the tooth. We determined the prevalence and predictors of periapical radiolucency in patients with cirrhosis and the association with systemic inflammation status and cirrhosis-related complications.

Methods: A total of 110 cirrhosis patients were consecutively enrolled. Periapical radiolucency was defined as the presence of radiolucency or widening of the periapical periodontal ligament space to more than twice the normal width. Predictors of periapical radiolucency and the association with systemic inflammation markers and cirrhosis-related complications were explored by univariable and multivariable logistic regression analyses.

Results: Periapical radiolucency was present in one or more teeth in $46 \%$ of the patients. Strong predictors were gross caries (odds ratio [OR] 3.12, 95\% confidence interval [CI] 1.43-6.79) and severe periodontitis (OR 3.98, 95\% CI 1.04-15.20). Also old age (OR 1.10, 95\% CI 1.01-1.19) and smoking (OR 3.24, 95\% CI 1.02-17.62) were predictors. However, cirrhosis etiology (alcoholic vs nonalcoholic) or severity (Model of End-Stage Liver Disease score) were not predictors. The patients with periapical radiolucency had higher C-reactive protein $(15.8 \mathrm{mg} / \mathrm{L}$ vs $8.1 \mathrm{mg} / \mathrm{L}, P=0.02)$ and lower albumin contents $(25 \mathrm{~g} / \mathrm{L}$ vs $28 \mathrm{~g} / \mathrm{L}, P=0.04)$ than those without. Furthermore, the patients with periapical radiolucency had a higher prevalence of cirrhosis-related complications such as ascites, hepatic encephalopathy, and/or variceal bleeding ( $46 \%$ vs $27 \%, P=0.05$ ).

Conclusion: Periapical radiolucency is often present as an element of poor oral health status and likely has an adverse clinical significance, which should motivate diagnostic and clinical attention to the findings.

Keywords: apical periodontitis, cirrhosis, C-reactive protein, inflammation status, MELD score, periapical radiolucency

\section{Introduction}

Periapical radiolucency is the descriptive term for radiographic changes which are most often due to apical periodontitis and radicular cysts, that is, inflammatory bone lesions around the apex of the tooth which develop if bacteria are spread from the oral cavity through a caries-affected tooth with necrotic dental pulp. ${ }^{1,2}$ Clinical signs and symptoms such as pain, tenderness, and swelling may occur in varying degrees, depending on the diagnosis. ${ }^{3}$

Although recent studies of cirrhosis patients have provided evidence for poor oral health and increased prevalence of periodontal diseases, ${ }^{4-7}$ which may accelerate the progression of the liver disease ${ }^{8}$ the prevalence of periapical radiolucency 
in these patients has so far been subjected to only a few studies..$^{5,9,10}$ It is thus unknown whether alcoholic cirrhosis and decompensation of the cirrhosis disease are associated with periapical radiolucency as some of these patients are generally more likely to have poor oral care habits and dental caries. ${ }^{7}$

Infection as a complication of cirrhosis is a frequent cause of increased morbidity and mortality. ${ }^{11,12}$ Periapical radiolucency due to apical periodontitis may contribute to this problem as it has been reported that apical periodontitis can precipitate a systemic inflammation activation in both healthy persons and patients with a variety of diseases. ${ }^{13,14}$ However, it remains unknown whether this is also true for cirrhosis patients.

Thus, the aim of this study was to determine the prevalence and identify the predictors of periapical radiolucency and to examine the association between periapical radiolucency and systemic inflammation status and cirrhosis-related complications in a cohort of patients with cirrhosis.

\section{Methods}

The study was conducted at Aarhus University Hospital, Denmark, between April 2013 and December 2015. The participating cirrhosis patients were consecutively recruited at the Department of Hepatology and Gastroenterology, which has a large local catchment population and also receives referred patients. The patient cohort was partly the same as previously presented using other data. ${ }^{15}$ Regardless of etiology and severity, all adult patients with an established diagnosis of cirrhosis who were able to give consent and to cooperate in a clinical and radiographic dental examination, and who had two or more teeth, were invited to participate. The diagnosis of cirrhosis was based on either biopsy and/ or clinical, biochemical, and ultrasonic findings. The examination was carried out with the understanding and written consent of each patient and according to the Declaration of Helsinki. The study was approved by the Central Denmark Region Committees on Health Research Ethics (number 1-10-72-128-12).

\section{Radiographic examination}

The periapical status was assessed using digital panoramic radiography. Three trained and experienced dental hygienists performed the panoramic radiography using a Planmeca ProMax 3D. The method of viewing the radiographies was standard. The images were examined in a room with controllable ambient lighting, using a computer with Planmeca Romexis software. The number of teeth present and the location and number of teeth having identifiable periapical lesions were recorded for each patient.

The periapical status of each tooth was assessed at 0 for normal: the periapical periodontal ligament space and the surrounding bone showed no alteration - and 1 for periapical radiolucency: the presence of radiolucency or widening of the periapical periodontal ligament space to more than twice the normal width. ${ }^{16}$

A senior resident in oral and maxillofacial surgery (author KS) with extensive clinical experience examined the radiographs after thorough training and calibration at the Department of Odontology, Aarhus University.

In connection with the radiographic examination, a clinical examination was performed by one of three dental hygienists at the Department of Hepatology and Gastroenterology, Aarhus University Hospital. Number of teeth, gross caries, that is, a form of caries with advanced dental decay that is easily detected clinically, ${ }^{17}$ plaque score, clinical probing depth, clinical attachment level, and bleeding on probing were registered to determine the dental status and the prevalence of severe periodontitis in the patients. Severe periodontitis was defined by having $\geq 2$ interproximal sites with clinical attachment loss $\geq 6 \mathrm{~mm}$ (not of the same tooth) and $\geq 1$ interproximal site with pocket depth $\geq 5 \mathrm{~mm} .{ }^{18} \mathrm{~A}$ detailed description of the examination is shown elsewhere. ${ }^{15}$

\section{Data collection}

Questions were asked or data were retrieved from the medical charts with respect to patients' age, sex, smoking habits, alcohol consumption, burden of comorbidity (Charlson Comorbidity Index), ${ }^{19}$ cirrhosis etiology, cirrhosis severity, current cirrhosis-related complications (ie, episodes of ascites, hepatic encephalopathy, and/or variceal bleeding), and oral care habits (ie, frequency of tooth brushing, frequency of dental visits, and feeling of dry mouth). Smoking status was classified as no smoking (if the patient had never smoked or was a former smoker) or as currently smoking. The patients' Model of End-Stage Liver Disease (MELD) score, C-reactive protein (CRP), and plasma albumin ( $\mathrm{P}$-albumin) were obtained from routine blood samples, and taken on the same day as the oral and radiographic examinations.

\section{Statistical analyses}

Univariable and multivariable logistic regression analyses were used to determine predictors associated with periapical radiolucency and to evaluate the association between the presence of periapical radiolucency and systemic 
inflammation status and cirrhosis-related complications. The candidate predictor variables were as follows: age, male sex (yes/no), smoking (yes/no), comorbidity, visit dentist annually (yes/no), gross caries, severe periodontitis (yes/no), alcoholic cirrhosis (yes/no), MELD score, current cirrhosisrelated complications (yes/no), CRP, and P-albumin. Beside the cirrhosis and systemic inflammation measures, the predictor variables were selected from those reported to be associated with periapical lesions and apical periodontitis, as described in previous studies. ${ }^{20}$ Continuous variables were entered into the analyses in untransformed form. Two multivariable, logistic regression analyses were performed. In the first, all the predictor variables were entered into one multivariable, logistic regression analysis and assessed simultaneously. In the second, a backward, stepwise logistic regression with $P>0.2^{21}$ was used to identify a small set of strong predictors and evaluate the association between systemic inflammation status/cirrhosis-related complications and periapical radiolucency. In all tests, a $P$-value of 0.05 or less was considered to be statistically significant. The data were analyzed using Stata version 12.0 (StataCorp LP, College Station, TX, USA).

\section{Results}

A total of 110 patients participated in the study. Their mean age was 59 years (range $39-82$ years), and $76 \%$ were men. Their demographic and clinical characteristics in relation to the presence of periapical radiolucency status are presented in Table 1.

The overall prevalence of periapical radiolucency in all the examined teeth was $4 \%$, and one or more teeth with periapical radiolucency (range 1-9) were found in $46 \%$ of the patients. A majority of the patients had only one (59\%) or two $(32 \%)$ teeth with periapical radiolucency.

There were no difference in age, sex, smoking status, alcohol use, comorbidity, cirrhosis etiology or severity, number of teeth, brushing frequency, and feeling of dry mouth in patients with periapical radiolucency compared with patients without. However, patients with one or more periapical radiolucencies attended the dentist less often than patients without periapical radiolucency (Table 1).

Table I Characteristics of the patient cohort

\begin{tabular}{|c|c|c|}
\hline Characteristics & Patients with periapical radiolucency & Patients without periapical radiolucency \\
\hline Number of patients & 51 & 59 \\
\hline Age (years) & $60(53-64)$ & $59(50-63)$ \\
\hline Female/male (\%) & $24 / 76$ & $45 / 55$ \\
\hline \multicolumn{3}{|l|}{ Smoking status (\%) } \\
\hline Yes/no & $51 / 49$ & $31 / 69$ \\
\hline \multicolumn{3}{|l|}{ Alcohol use (\%) } \\
\hline Yes/no & $44 / 56$ & $25 / 75$ \\
\hline \multicolumn{3}{|l|}{ Charlson Comorbidity Index (\%) } \\
\hline $3+$ & 4 & 0 \\
\hline 2 & 7 & 13 \\
\hline I & 27 & 24 \\
\hline 0 & 63 & 63 \\
\hline \multicolumn{3}{|l|}{ Oral care habits (\%) } \\
\hline Brushing teeth twice daily & 32 & 49 \\
\hline Visiting dentist annually & 24 & 46 \\
\hline Dry mouth (nearly always or always) & 25 & 24 \\
\hline \multicolumn{3}{|l|}{ Oral measures } \\
\hline Number of teeth & $22(17-26)$ & $26(16-28)$ \\
\hline Gross caries (\%) & 46 & 10 \\
\hline Severe periodontitis (\%) & 66 & 39 \\
\hline \multicolumn{3}{|l|}{ Cirrhosis etiology (\%) } \\
\hline Alcohol & 76 & 65 \\
\hline Cryptogenic & 17 & 8 \\
\hline Autoimmune or cholestatic & 7 & 27 \\
\hline \multicolumn{3}{|l|}{ Cirrhosis severity } \\
\hline MELD score & II (8-15) & II (7-15) \\
\hline Patients with current cirrhosis-related complications (\%) & 46 & 27 \\
\hline \multicolumn{3}{|l|}{ Inflammation status } \\
\hline C-reactive protein (mg/L) & $15.8(6.9-40.3)$ & $8.1(5.6-24.0)$ \\
\hline P-albumin (g/L) & $25(22-31)$ & $28(25-35)$ \\
\hline
\end{tabular}

Note: Data are presented as number, percentage, or median and interquartile range. Abbreviation: MELD, Model of End-Stage Liver Disease. 
Table 2 Univariable and multivariable logistic regression analyses of the association between the predictor variables and the outcome variables in periapical radiolucency

\begin{tabular}{|c|c|c|c|c|c|c|}
\hline \multirow[t]{2}{*}{ Variables } & \multicolumn{2}{|l|}{ Univariable } & \multicolumn{2}{|c|}{ Multivariable } & \multicolumn{2}{|c|}{ Stepwise multivariable } \\
\hline & Odds ratio & $95 \% \mathrm{Cl}$ & Odds ratio & $95 \% \mathrm{Cl}$ & Odds ratio & $95 \% \mathrm{Cl}$ \\
\hline Age, per year increase & 1.03 & $0.99-1.07$ & 1.09 & $1.00-1.20$ & 1.10 & $1.01-1.19$ \\
\hline Male sex (yes/no) & 2.53 & $1.02-6.26$ & 1.71 & $0.50-7.21$ & & \\
\hline Smoking (yes/no) & 2.31 & $0.97-5.48$ & 3.31 & $1.09-16.63$ & 3.24 & $1.02-17.62$ \\
\hline Comorbidity & 1.04 & $0.60-1.79$ & 1.13 & $0.5 \mathrm{I}-2.34$ & & \\
\hline Visiting dentist annually (yes/no) & 0.36 & $0.15-0.90$ & 0.87 & $0.23-3.24$ & & \\
\hline Gross caries & 2.63 & $1.37-5.04$ & 2.99 & $1.45-6.17$ & 3.12 & $1.43-6.79$ \\
\hline Severe periodontitis (yes/no) & 3.05 & $1.28-7.23$ & 3.39 & $1.00-16.70$ & 3.98 & $1.04-15.20$ \\
\hline Alcoholic cirrhosis (yes/no) & 1.55 & $0.6 \mathrm{I}-3.94$ & 1.05 & $0.31-1.29$ & & \\
\hline MELD score & 1.01 & $0.95-1.08$ & 1.00 & $0.90-1.11$ & & \\
\hline Current cirrhosis compilations (yes/no) & 2.39 & $1.58-5.78$ & 2.34 & $1.54-34.99$ & 2.90 & $1.91-32.68$ \\
\hline CRP, per $\mathrm{mg} / \mathrm{L}$ increase & 1.02 & $1.01-1.04$ & 1.02 & $1.01-1.06$ & 1.02 & $1.01-1.06$ \\
\hline P-albumin, per $\mathrm{g} / \mathrm{L}$ increase & 0.94 & $0.89-0.99$ & 0.96 & $0.89-0.99$ & 0.96 & $0.89-0.99$ \\
\hline
\end{tabular}

Note: Statistically significant associations are shown in bold.

Abbreviations: $\mathrm{Cl}$, confidence interval; CRP, C-reactive protein; MELD, Model of End-Stage Liver Disease.

Oral health markers in the form of gross caries (odds ratio [OR] 3.12, 95\% confidence interval [CI] 1.43-6.79) and severe periodontitis (OR 3.98, 95\% CI 1.04-15.20) were predictors of periapical radiolucency. The same was found for old age (OR 1.10, 95\% CI 1.01-1.19) and smoking (OR 3.24, 95\% CI 1.02-17.62). Alcoholic versus nonalcoholic cirrhosis and MELD score were not associated with periapical radiolucency (Table 2).

The median CRP concentration was $15.8 \mathrm{mg} / \mathrm{L}$ in the group of patients with one or more teeth with periapical radiolucency compared with $8.1 \mathrm{mg} / \mathrm{L}$ in the patients without periapical radiolucency $(P=0.02)$. Likewise, $\mathrm{P}$-albumin was slightly lower in the patients with periapical radiolucency than in those without ( 25 vs $28 \mathrm{~g} / \mathrm{L}, P=0.04$ ). Furthermore, the patients with periapical radiolucency had a higher prevalence of current cirrhosis-related complications such as ascites, episodes of hepatic encephalopathy, and/or variceal bleeding ( $46 \%$ vs $27 \%, P=0.05$ ) (Table 2 ). Figure 1 shows a panoramic radiography of periapical radiolucency.

\section{Discussion}

In this study, nearly half of the patients with cirrhosis had periapical radiolucency, independently of alcoholic versus nonalcoholic etiology and cirrhosis severity. Gross caries, periodontitis, age, and smoking emerged as predictors. The presence of periapical radiolucency was associated with signs of systemic inflammation activation and with more frequent cirrhosis-related complications.

Periapical radiolucency may be caused by a variety of diseases, with apical periodontitis and radicular cysts being the most common and cementoblastoma and periapical cemental dysplasia being less frequent. Still, it is not

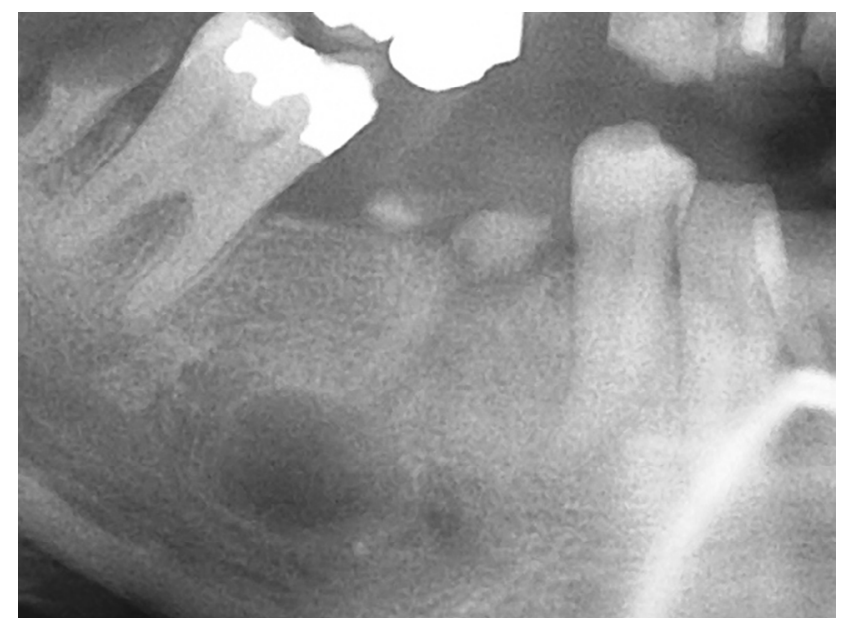

Figure I Radiograph of right mandibular second premolar showing gross caries and periapical radiolucency.

possible to translate the radiographic findings to a specific diagnosis. ${ }^{22,23}$ In the Scandinavian countries, the reported prevalence of apical periodontitis ranges from $1.5 \%$ to $3.4 \%$ at tooth level and $30 \%-50 \%$ at individual level. ${ }^{20,24,25}$ Lins et $\mathrm{a}^{9}$ have found radiographic signs of periapical lesions in $48 \%$ of their cirrhosis cohort, which is in line with our findings, but below the $79 \%$ found in a study of liver transplant candidates. ${ }^{10}$ This might suggest that the frequency of periapical radiolucency that we report in our cirrhosis patients is not higher than in the background population. However, it should be taken into account that the criteria for periapical radiolucency vary markedly among the available studies. In our study, periapical radiolucency was diagnosed from digital panoramic radiographs using preestablished criteria ${ }^{16}$ rather than the "periapical index", ${ }^{10,26}$ which is used in population studies and which highly overestimates the prevalence of the lesions. ${ }^{27}$ 
The registration of periapical radiolucency was collected from panoramic radiographs because of their suitability for the clinical setting, although this method may be considered as a less sensitive diagnostic tool compared with full-mouth radiographs. However, the authors have argued that the use of panoramic radiography in epidemiological studies is acceptable, and they found a high specificity and sensitivity to the detection of periapical pathology compared with fullmouth radiographs..$^{28}$ The risk of underestimating the score of periapical lesions on panoramic radiographs is thus most likely low, and the overall validity of evaluating the periapical conditions is therefore reliable. ${ }^{29}$

No association emerged between periapical radiolucency and cirrhosis etiology. This is unexpected as alcoholic cirrhosis is often linked to a lifestyle of self-neglect, leading to a large number of carious teeth and extensive periodontal disease, ${ }^{7}$ and as autoimmune liver disorders such as primary biliary cirrhosis are related to Sjögren's syndrome, which may cause rapid tooth decay. ${ }^{4}$ However, no difference was found in the feeling of dry mouth between the patients with or without periapical radiolucency.

Likewise, in this study, the MELD score was not a predictor for periapical radiolucency, although a recent study has reported a correlation between oral infections and accelerated progression of liver disease measured by the MELD score. ${ }^{8}$ However, our findings indicate that it is the cirrhosis and the associated poor oral health status that predispose to periapical radiolucency rather than the etiology or severity of cirrhosis.

Older age and smoking were predicatively associated with periapical radiolucency in our cirrhosis patients. The importance of these factors in the general population is well documented. ${ }^{20,30}$ Likewise, oral care habits have been associated with periapical lesions. ${ }^{20}$ This is consistent with our study where an association was found between annual visits to the dentist and periapical radiolucency in the univariable analysis.

The predictive value of gross caries and severe periodontitis is mechanistically relevant as both lesions are main gateways for bacteria entering into the surrounding tissue and thereby providing access to the bloodstream. ${ }^{31,32}$

Previous studies report that apical periodontitis may lead to systemic inflammation activation. ${ }^{14,33,34}$ In accordance with this finding and the assumption that most of our cases of periapical radiolucency were due to apical periodontitis, it is noteworthy that the cirrhosis patients with radiolucent lesions had a double level of CRP and a slightly lower level of P-albumin than those without. This has not earlier been shown in patients with cirrhosis, but it may evidently have untoward consequences in the clinical course of the cirrhosis disease which is often fraught with infectious episodes as major precipitating factors for the well-known cirrhosis complications, thus contributing to the high morbidity and mortality of the disease. ${ }^{12}$ The level of systemic inflammation activation as judged from CRP and P-albumin was not very marked in the patients with periapical radiolucency. However, similar to intestinal translocation, the dental infections may well be a whole-time, latent "internal" source of bacterial contamination of the bloodstream, resulting in low-grade inflammation, which is known to be a source of systemic complications. ${ }^{35}$ The present findings of more cirrhosis-related complications among patients with periapical radiolucency are in line with this view, and it may imply that more clinical attention should be directed toward oral examination and clinical care.

There are limitations to this study. First, the crosssectional study design provides information only on associations among the study variables and not on causality. Second, the observed associations could to some extent be due to confounding variables, such as unmeasured biological variables, or socioeconomic conditions, although other studies indicate that these do not have obvious implications for periapical disease. ${ }^{36}$ Finally, no intra-examiner reliability testing was carried out. It was important that the participating patients were not disturbed more than absolutely necessary, and as the radiographs were taken by a different department it hampered the recall of the patients for intra-examiner testing. This is problematic and impairs the reliability of the examinations, even though it has been argued that the benefits of intra-examiner testing are limited ${ }^{37}$ However, a high reliability can be expected due to the fact that the examiner is a senior resident with extensive clinical experience. Furthermore, the examiner related his findings to written criteria throughout the whole evaluation.

In conclusion, the present results contribute new information to the sparse knowledge of periapical disease. It was observed that almost half of the patients presented periapical radiolucency. Neither cirrhosis etiology nor severity was predictors. However, periapical radiolucency was associated with systemic inflammation activation and clinical cirrhosis complications. Further studies are needed to confirm and expand these findings and to evaluate whether improved clinical care of oral health may improve the disease burden related to inflammation and infection in patients with cirrhosis. 


\section{Acknowledgments}

The authors would like to thank dental hygienists Nanna Jensen, Natasja Nielsen, and Susanne Hedegaard for performing clinical examinations and the Department of Oral and Maxillofacial Surgery, Aarhus University Hospital, Aarhus, Denmark, for performing the radiographic examinations.

This work is supported by grants from Aarhus University Hospital, Aase and Ejnar Danielsen's Foundation, A.P. Møller Foundation, Central Denmark Region Foundation for Health Research, and Novo Nordisk Foundation.

\section{Disclosure}

The authors report no conflicts of interest in this work.

\section{References}

1. Ricucci D, Mannocci F, Ford TR. A study of periapical lesions correlating the presence of a radiopaque lamina with histological findings. Oral Surg Oral Med Oral Pathol Oral Radiol Endod. 2006;101(3):289-394.

2. Stashenko P. Aetiology and pathogenesis of pulpitis and apical periodontitis. In: Ørstavik D, Ford P, editors. Essential Endodontology. Oxford: Blackwell Science; 1998:42-67.

3. Bukmir RP, Grgic MJ, Brumini G, Spalj S, Pezelj-Ribaric S, Prso IB. Influence of tobacco smoking on dental periapical condition in a sample of Croatian adults. Wien Klin Wochenschr. 2016;128(7-8):260-265.

4. Grønkjær LL, Vilstrup H. Oral health in patients with liver cirrhosis. Eur J Gastroenterol Hepatol. 2015;27(7):834-839.

5. Helenius-Hietala J, Meurman JH, Höckerstedt K, Lindqvist C, Isoniemi $\mathrm{H}$. Effect of the aetiology and severity of liver disease on oral health and dental treatment prior to transplantation. Transpl Int. 2012;25(2):158-165.

6. Oettinger-Barak O, Barak S, Machtei EE, Ardekian L, Baruch Y, Peled M. Periodontal changes in liver cirrhosis and post-transplantation patients. I: clinical findings. J Periodontol. 2001;72(9):1236-1240.

7. Novacek G, Plachetzky U, Pötzi R, Lentner S, Slavicek R, Gangl A, Ferenci P. Dental and periodontal disease in patients with cirrhosis - role of aetiology of liver disease. J Hepatol. 1995;22(5):576-582.

8. Åberg F, Helenius-Hietala J, Meurman J, Isoniemi H. Association between dental infections and the clinical course and chronic liver disease. Hepatol Res. 2014;44(3):349-353.

9. Lins L, Bittencourt PL, Evangelista MA, et al. Oral health profile of cirrhotic patients awaiting liver transplantation in the Brazilian Northeast. Transplant Proc. 2011;43(4):1319-1321.

10. Castellanos-Cosano L, Machuca-Portillo G, Segura-Sampedro JJ, Torres-Lagares D, López-López J, Velasco-Ortega E, Segura-Egea JJ. Prevalence of apical periodontitis and frequency of root canal treatments in liver transplant candidates. Med Oral Patol Oral Cir Bucal. 2013;18(5):773-779.

11. Preda S, Trifan A, Girleanu I, Stanciu C, Cojocariu C. Infectious complications in patients with liver cirrhosis. Rev Med Chir Soc Med Nat Iasi. 2014;118(3):590-597.

12. Jalan R, Fernandez J, Wiest R, et al. Bacterial infections in cirrhosis: a position statement based on the EASL Special Conference 2013. J Hepatol. 2014;60(6):1310-1324.

13. Vidal F, Fontes TV, Marques TVF, Goncalves LS. Association between apical periodontitis lesions and plasmatic levels of C-reactive protein, interleukin 6 and fibrinogen in hypertensive patients. Int Endod J. 2015;24:1-9.
14. Gomes MS, Blattner TC, Santana Filho M. Can apical periodontitis modify systemic levels of inflammatory markers? A systematic review and meta-analysis. $J$ Endod. 2013;39(10):1205-1217.

15. Grønkjær LL, Holmstrup P, Schou S, Kongstad J, Jepsen P, Vilstrup H. Periodontitis in cirrhosis. World J Gastroenterol. Epub 2016 July 13.

16. De Moor RJ, Hommez GM, De Boever JG, Delme KI, Mertens GE. Periapical health related to the quality of root canal treatment in a Belgian population. Int Endod J. 2000;33(2):113-120.

17. Hillson S. Teeth. 2nd ed. UK: Cambridge University Press; 2005.

18. Page RC, Eke PI. Case definitions for use in population-based surveillance of periodontitis. J Periodontol. 2007;78(7 Suppl):1387-1399.

19. Quan H, Li B, Couris CM, et al. Updating and validating the Charlson comorbidity using data from 6 countries. Am J Epidemiol. 2011; 173(6):676-682.

20. Kirkevang LL, Wenzel A. Risk indicators for apical periodontitis. Community Dent Oral Epidemiol. 2003;31(1):58-67.

21. Budtz-Jørgensen E, Keiding N, Grandjean P, Weihe P. Confounder selection in environmental epidemiology: assessment of health effects of prenatal mercury exposure. Ann Epidemiol. 2007;17(1):27-35.

22. Razavi SM, Kiani S, Khalesi S. Periapical lesions: a review of clinical, radiographic, and histopathologic features. Avicenna J Dent Res. 2014;6:e19435.

23. Peters E, Lau M. Histopathologic examination to confirm diagnosis of periapical lesions: a review. J Can Dent Assoc. 2003;69(9):598-600.

24. Odesjo B, Hellden L, Salonen L, Langeland K. Prevalence of previous endodontic treatment, technical standard and occurrence of periapical lesions in a randomly selected adult, general population. Endod Dent Traumatol. 1990;6(6):265-272.

25. Eriksen HM, Bjertness E, Orstavik D. Prevalence and quality of endodontic treatment in an adult urban population in Norway. Endod Dent Traumatol. 1988;4(3):122-126.

26. Ørstavik D, Kerekes K, Eriksen HM. The periapical index: a scoring system for radiographic assessment of apical periodontitis. Endod Dent Traumatol. 1986;2(1):20-34.

27. Gomes MS, Hugo FN, Hilgert JB, et al. Apical periodontitis and incident cardiovascular events in the Baltimore Longitudinal Study of Ageing. Int Endod J. 2016;49(4):334-342.

28. Ahlqwist M, Halling A, Hollender L. Rational panoramic radiography in epidemiological studies of dental health. Comparison between panoramic radiographs and intraoral full mouth surveys. Swed Dent J. 1986;10(1-2):73-84.

29. Molander B, Ahlqwist M, Gröndahl HG. Panoramic and restrictive intraoral radiography in comprehensive oral radiographic diagnosis. Eur J Oral Sci. 1995;103(4):191-198.

30. Walter C, Rodriguez FR, Taner B, Hecker H, Weiger R. Association of tobacco use and periapical pathosis - a systemic review. Int Endod J. 2012;45(12):1065-1073.

31. Kirkevang LL, Ørstavik D, Bahrami G, Wenzel A, Vaeth M. Prediction of periapical status and tooth extraction. Int Endod J. 2015;1:1-10.

32. Jansson L, Ehnevid H, Lindskog S, Blomlöf L. Relationship between periapical and periodontal status: A clinical retrospective study. J Clin Periodontol. 1993;20(2):117-123.

33. Van der Waal SV, Lappin DF, Crielaard W. Does apical periodontitis have systemic consequences? The need for well-planned and carefully conducted studies. Br Dent J. 2015;218(9):513-516.

34. Cotti E, Dessi C, Piras A, Mercuro G. Can a chronic dental infection be considered a cause of cardiovascular disease? a review of the literature. Int J Cardiol. 2011;148(1):4-10.

35. Belstrøm D, Damgaard C, Nielsen CH, Holmstrup P. Does a causal relation between cardiovascular disease and periodontitis exist? Microbes Infect. 2012;14(5):411-418.

36. Frisk F, Hakeberg M. Socio-economic risk indicators for apical periodontitis. Acta Odontol Scand. 2006;64(2):123-128.

37. Reit C. The influence of observer calibration on radiographic periapical diagnosis. Int Endod J. 1987;20(2):75-81. 


\section{Publish your work in this journal}

Hepatic Medicine: Evidence and Research is an international, peerreviewed, open access journal covering all aspects of adult and pediatric hepatology in the clinic and laboratory including the following topics: Pathology, pathophysiology of hepatic disease; Investigation and treatment of hepatic disease; Pharmacology of drugs used for the treatment of hepatic disease. Issues of patient safety and quality of care will also be considered. The manuscript management system is completely online and includes a very quick and fair peer-review system, which is all easy to use. Visit http://www.dovepress.com/testimonials.php to read real quotes from published authors.

Submit your manuscript here: https://www.dovepress.com/hepatic-medicine-evidence-and-research-journal 